\title{
Luz: From Light to Darkness: Lessons learned from the solar system
}

\author{
Joseph E. Kasser \\ The Right Requirement Ltd \\ 50 Crane Way \\ Cranfield, Beds, MK43 OHH, England \\ Joseph.Kasser@incose.org
}

Copyright $\odot 2008$ by Joseph E. Kasser. Used by INCOSE with permission for the INCOSE 2008 Symposium.

\begin{abstract}
In teaching systems engineering the relationship between functions, physical decomposition and requirements during the process of defining, designing and developing the system, has been difficult to get across to the students. While trying to improve the learning process, an explanation of the relationship between functions, physical decomposition and requirements during the process of defining, designing and developing the system based on a modification of the Functions Requirements Answers and Test (FRAT) views of a system (Mar, 1994) was tried on undergraduate students at the University of South Australia in 2006-2007 with positive results (Kasser, et al., 2007). This paper documents the LuZ SEGS-1 system design process in the form of the FRAT views demonstrating the intertwined relationships between requirements, functions and their allocation to components at a lower level of system decomposition. The paper provides examples of alternative choices, discusses them and documents the choice with the reasons for selection. Several lessons learned from the project are also provided.
\end{abstract}

\section{Introduction}

The systems engineering process contains the sequence of activities in which as a response to an identified need, a conceptual system is defined, designed, developed and placed into service. However, in teaching systems engineering the relationship between functions, physical decomposition and requirements during the process of defining, designing and developing the system, has been difficult to get across to the students.

While trying to improve the learning process, an explanation of the relationship between functions, physical decomposition and requirements during the process of defining, designing and developing the system based on a modification of the Functions Requirements Answers and Test (FRAT) views of a system (Mar, 1994) was tried on undergraduate students at the University of South Australia in 2006-2007. During the learning process, the questions they asked indicated that they were demonstrating a better grasp of the concepts than had been demonstrated by postgraduate students in earlier courses (Kasser, et al., 2007).

This paper uses the adapted FRAT as a frame in which to describe the relationship between functions, physical decomposition and requirements using as an example the definition, design and development project for the control and electronics part of the LuZ solar electrical power generating system (SEGS-1) in 1981-1983 (Kasser, 1984). The paper also provides some lessons learned from the project.

\footnotetext{
${ }^{1}$ The writing of this paper was made possible by a grant from The Leverhulme Trust to Cranfield University.
} 
Module 4

Accepted to the18th INCOSE International Symposium, Utrecht, Holland, 2008

\section{The FRAT approach}

Functions Requirements Answers and Test (FRAT) (Mar, 1994; Mar and Morais, 2002) was introduced as four views of a system. However, the "A" and " $T$ " in FRAT are also useful for investigation of conceptual alternatives which provides a useful perspective, namely:

- Functions which define what functions the solution must perform (operational and functional perspectives).

- Requirements which define how well each function must be performed (quantitative perspective).

- Answers which use the scientific perspective to search not only for one solution, but for a better solution as well and manage risk associated with that solution (descriptive perspectives). The answer element concludes with a decision as to which of the alternative answers identified as providing a solution to the problem is to be selected for implementation.

- Tests which demonstrate that the selected solution performs the needed functions according to the requirements. The scientific perspective is used to create the tests and the quantitative perspective to determine the expected test results.

In use, FRAT is not performed in a sequential manner. It is instead performed in an iterative series-parallel manner in which each of the four elements is both an input to and an output of the others as shown in Figure 1. As an example, the findings in one element may affect the contents of another. The use of FRAT will now be illustrated in the case of the systems development process for the world's first commercial solar electrical power generating system (SEGS-1) (Kasser, 1984; Kasser, 2001; Kasser and Palmer, 2005).

\section{Background to the case study}



Figure 1The FRAT approach

The LuZ Group, a start-up joint Israel-American venture

defined, designed, developed, installed and operated SEGS-1 in 1981-1983. At the design time, as the first of its kind, SEGS-1 initially only existed as a vague concept and met (Donaldson and Siegel, 1997)'s definition of a (very) high risk project. SEGS-1 was installed in the Mojave Desert in California and the Research and Development was in performed in Jerusalem. SEGS-1 was intended to generate electrical power from the sun by focussing the sun's rays on about 600 parabolic mirror trough reflector collectors each about 40 meters long. The operation of each parabolic trough reflector would be monitored and controlled by a microprocessor based local controller (LOC). Each LOC would control a motor that would position the parabolic mirror, receive information about the angle of elevation of the mirror and the temperature of the oil in the pipe positioned at the focus of the trough. Oil would be pumped through the piping, and as long as the LOC would keep the reflector pointed at the sun within an accuracy of \pm 0.2 degrees, the oil would be heated. The hot oil would be pumped around the field and into a heat exchanger to generate steam. The steam would then drive a turbine that generated up to 15 Megawatts of electrical power. Although it would be a complicated system, it would still have a conversion efficiency of about $40 \%$, greater than any alternative method of harnessing solar energy at the time. 
Module 4

Accepted to the18th INCOSE International Symposium, Utrecht, Holland, 2008

\section{At the system level}

Consider the system from various perspectives (Kasser, 2008), the details and insights that emerge include ${ }^{2}$ :

Big picture perspective. The big picture perspective includes:

1. SEGS-1 is the containing or meta-system.

2. The system under consideration/discussion is limited to the control and electronics system (CES) for the array of solar collectors within SEGS-1.

3. The CES is a system in itself, and at the same time is a subsystem of SEGS-1.

4. The oil pumping and heat transfer elements of the solar field, and the array of mirrors, are outside the CES. They interface to the CES electronically via the oil temperature sensors and mechanically via the motors.

5. The solar collection array is to be located in an area the size of a football field.

6. There is an adjacent totally decoupled system, an experimental heliostat solar power generating field which may provide a false sun via the reflection from the central tower and the LOCs may lock on to it and fail to follow the sun.

7. There is no space in the development facility to build and test a large array of LOCS prior to deployment half way around the world. The design and test programs will have to compensate for this constraint.

Operational perspective. The CES operates automatically daily as long as there is no major cloud cover. Manual operation takes place in certain scenarios. Each and every mirror can only move in one axis - elevation. The azimuth is fixed approximately north-south.

Functional perspective. The mirrors deploy to track the sun in the morning as soon as the sun is high enough to generate positive power, track the sun during the day and stow in the evening. The CES performs the following functions:

- Stowed. The mirrors are at rest in an upside down position. This minimises dirt collection and sand abrasion of their surfaces which reduce the reflection coefficient and hence the efficiency of the system. Since the mirrors also act as radiators when not acting as heaters, stowing the mirror minimises heat loss to outer space.

- Deploying. The mirrors are moving up from the stowed position to begin to track (follow) the sun.

- Tracking. The mirrors follow the sun across the sky keeping the oil pipe at the focus, allowing the sun to heat the oil.

- Stowing. The mirrors are returning to the stow position at the end of the day.

- Manual movement. The mirrors are moving as commanded by the operator.

- Idle. The mirrors are stationary.

- Data collection, storage and reporting. The system generates, stores and reports information about the positions of the mirrors, and temperatures of the oil at their foci.

Structural perspective. The insight from the structural perspective includes that even though there are about six hundred LOCs, since each LOC will be identical to the others, there are really only four subsystems. These are the central control station (CS), the LOCs, the interconnecting network between the CS and the LOCs, and the power distribution system.

\footnotetext{
${ }^{2}$ This is not meant to be a complete treatment at the system level.
} 


\section{Module 4}

Accepted to the18th INCOSE International Symposium, Utrecht, Holland, 2008

Generic perspective. The insights provided by this perspective include:

1. The CES architecture is similar to a constellation of low earth orbiting satellites and a control station. This allows spacecraft telemetry tracking and control techniques to be considered as design options.

2. The mirror positioning function is similar to a satellite ground station positioning function.

3. Short duration loss of insolation due to intermittent clouds is similar to the loss of synchronizing pulses in an analogue TV signal, so a flywheel technique could be employed to compensate.

Continuum perspective. There are at least three design choices,

1. One end of the continuum, in which a smart CS manages the entire array as well as collecting and storing information about the CES,

2. the other end of the continuum in which a dumb CS collects and stores information about the system while the LOCs perform the mirror management functions, and

3. a mixture of the previous two, namely somewhere along the continuum.

Temporal perspective. This perspective indicates that the efficiency of SEGS-1 is expected to reduce over time due to physical effects in subsystems adjacent to the CES (such as loss of vacuum in the oil pipes). While SEGS-1 can be maintained after hours, it is undesirable (extra operating cost) and the identification and replacement of a failed component should be quick. It would also be desirable for the CES to be able to predict failing vacuums in the oil pipes so that replacements could be scheduled.

Quantitative perspective. This perspective shows the following issues.

1. Feasibility study calculations have shown that each 40 Meter long mirror must be pointed at the sun with an accuracy of \pm 0.2 degrees.

2. The CES uses AC power to move the mirrors and power the LOCs. To be practical, SEGS-1 must put more power into the electrical power grid than it takes out.

3. The more power it can produce, the greater the revenue to the investors who will own SEGS-1.

Scientific perspective. Alternative candidate solutions for the CS are minicomputer or microcomputer based.

\section{FRAT at the system level}

Applying FRAT at the CES system level we have ${ }^{3}$ :

Functions. The control function includes the following:

- Deploy the entire array of mirrors when the power to be generated by SEGS-1 is more than the power to be used.

- Track when and while the sun shines; idle for periods of cloud cover.

- Stow the array when the power to be generated by SEGS-1 is less than the power to be used.

- Gather, display and store status information about the operation of SEGS-1.

\footnotetext{
${ }^{3}$ Note there are meta system considerations as well in the discussion since they affect the control functions.
} 
Module 4

Accepted to the18th INCOSE International Symposium, Utrecht, Holland, 2008

Requirements. There are two performance requirements on the CES namely ${ }^{4}$ :

1. In operation, SEGS-1 shall generate more power than it uses.

2. SEGS-1 shall generate the maximum possible amount of power each day.

Answers. The key to meeting the requirements is the accuracy of positioning the mirrors. Calculations have shown that the derived requirement to be levied on the mirror pointing function is to point the mirror at the sun with an allowable offset of only \pm 0.2 degrees. The following alternative solution options for meeting this requirement were considered:

1. A tight control loop which would depend on accurate system information (tight tolerances) about the longitude, latitude, and mirror alignment with respect to north of the field; angle of sun sensor with respect to mirror axis, and other pertinent parameters for each mirror.

2. A sloppy control loop which would not require the tight tolerances of the previous option, with self-regulating components to maintain the system in the steady tracking state.

Some of the factors affecting the choice were as follows ${ }^{5}$ :

- While one LOC had the capability to control more than one mirror, the system architecture was simpler if each LOC only had to interface to a single mirror. In addition, the embedded software would have fewer instructions.

- The amount of functionality in the LOC microprocessor was yet to be determined.

- The sun seems to travel across the sky at a rate of 15 degrees an hour, or one degree in four minutes. The mirrors move slowly enough that the position control algorithm could be located in either the LOCs or in the central station.

- The quantitative perspective shows that assuming that each sun-sensor has a slightly different offset when installed on its mirror, if the position control algorithm (CES function) is located in the CS (physical component), it will have to compute the desired elevation angles for each mirror in the entire array and update the LOCs within 24 seconds (requirement on CS). This means that the CS and LOC processor software cycle times, the network data rates, message lengths and other parameters will have to be determined to ensure that the CES can meet the performance requirement.

- If the position control algorithm (CES function) is located in the LOC (physical component) number of instructions in the software will have to be estimated to ensure that the microprocessor cycle time is fast enough (requirement on LOC) to perform the computations.

- The angle between the array of mirrors and due north is difficult to determine to decimal point accuracy.

The functional analysis was first performed for a single function in the CS controlling all the

\footnotetext{
${ }^{4}$ These requirements have not been stated in numerical terms. The feasibility study has shown that the system can potentially generate 15 Megawatts of power. However, the power it uses to do that will be a function of the motors, the power distribution losses, etc. that were unknown at the time the requirements were stated. Stating them as goals in this manner means that once the design has been made, the 'deploy' and 'stow' commands can be based on calculations or on the amount of solar insolation.

${ }^{5}$ There were other decisions to be made that have not been included in this extract. One such decision early in the system design phase was should one LOC control a single mirror or should it control several mirrors? And if so, how many?
} 


\section{Module 4}

Accepted to the18th INCOSE International Symposium, Utrecht, Holland, 2008

LOCs and as shown below was found to be problematic with the microprocessor solution. However, instead of choosing the minicomputer alternative, an innovative partitioning approach was then postulated (scientific perspective) coming from the generic perspective on the similarity between the CES and a constellation of satellites. This physical allocation split the functionality between the LOC and the CS (with minimal coupling) enabling the microprocessor based CS solution.

The design decision was to develop a physical architecture in which one LOC controlled one mirror, and use the sloppy algorithm using a self-regulating approach; partitioning the control system functionality such that the responsibility for making the decision to deploy or stow resided in the CS, but the position control function of each mirror was the responsibility of its LOC.

This approach eliminated many of the calculations on the data rates and tolerances that would otherwise have been required. The only serious technical risk associated with this choice was that the chosen microprocessor cycle time might not be sufficient.

Test. Previous experience with microprocessor-based satellite ground station antenna controllers indicated that this risk had a very low probability of occurrence. Subsequent breadboarding verified that the LOC could do the task.

\section{At the subsystem level}

The functionality and requirements for the CES are now expanded at the subsystem level. This process contains steps in which options are determined and choices made. Sometimes tradeoffs have to be made between the locations of functionality, that is, to which part of which subsystem the functionality to meet a specific CES requirement is allocated.

A selection of the various physical choices to be made in allocating CES functionality to the physical subsystems, namely designing the CS, network and power distribution subsystems is shown in Figure $2^{6}$. These choices were:

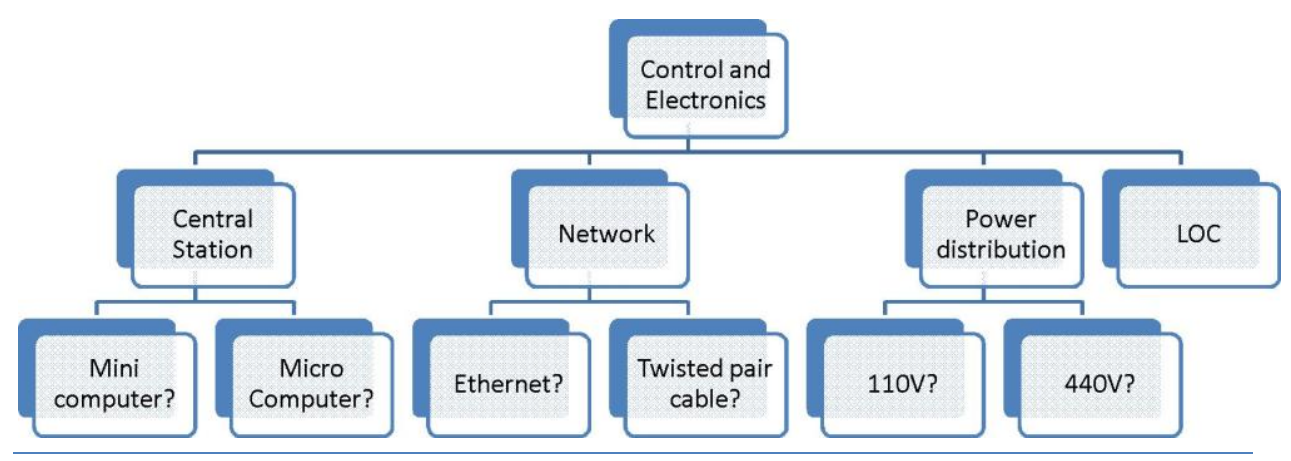

Figure 2 Part of the design choices at the subsystem level

3. CS. The physical alternatives were to use a mini- or micro-computer.

4. The Network. The physical alternatives were to use an Ethernet or twisted pair shielded wire.

5. The Power distribution. The alternatives were to distribute power to the LOCs at $110 \mathrm{~V}$, $240 \mathrm{~V}$ or $440 \mathrm{~V}$ using single or three phases.

${ }^{6}$ An alternative depiction is a decision tree. 
Module 4

Accepted to the18th INCOSE International Symposium, Utrecht, Holland, 2008

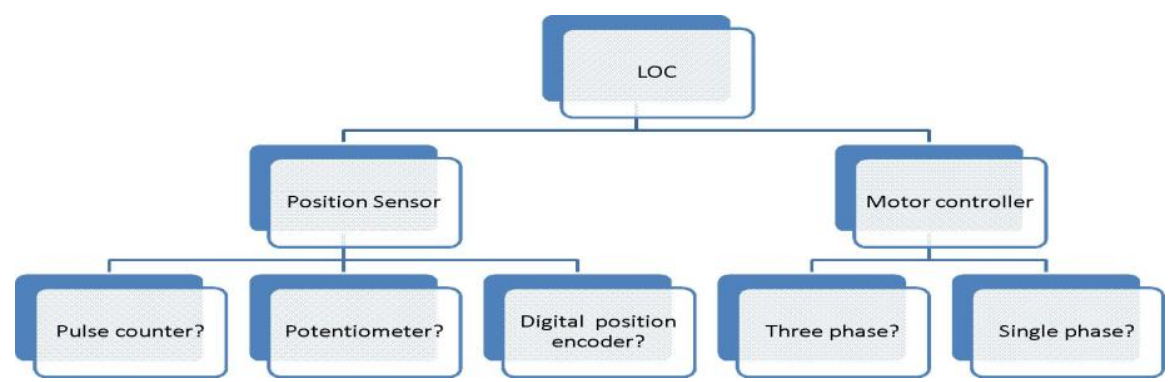

Figure 3 Part of the design choices for the LOC

6. The LOC. The LOC was the most complex subsystem. Some of the physical alternatives are shown separately in Figure 3 to keep the figures simple.

Consider the application of FRAT to the network, CS, power distribution and LOC subsystems in making those choices.

FRAT applied to the CS. Applying FRAT at the subsystem level to the CS we had:

Functions. The functions include:

1. Automatically deploying and stowing the LOCs at the appropriate times of day.

2. Acting as an operator interface to the array of mirrors allowing the operator to command and interrogate the LOCs.

3. Displaying CES status information.

4. Storing CES status information on a 10 Megabyte hard drive, offloading the data on magnetic media for archival storage offsite should the function be desired by the system developer or customer in the future ${ }^{7}$.

Requirements. The requirements inherited from the CES level included:

1. Determination of sun elevation angle for the field throughout the day.

2. Commanding and interrogating the LOCs individually or en-masse.

3. Displaying CES status information in a user friendly manner (location on the control screen, font size, colour, etc.)

4. Sounding alarms when various conditions exist.

Requirements on the CS to display information in various colours depending on the states of the CES and sounding the alarms are ergonomic requirements, not functional and performance. They are inherited from the class of system to which the CS belongs.

There were no detailed requirements for the actual operator commands and display of information. This functionality evolved in a rapid prototyping environment; the designers and systems engineer evolved the command and displays starting with a concept of "what and how" and working with the software to modify it as experience was gained. Ideas from the adjacent Heliostat system were considered and included as appropriate. The requirements were written down eventually for the purpose of documenting the actual functionality achieved rather than documenting what functionality would have to be achieved ${ }^{8}$.

\footnotetext{
${ }^{7}$ The hardware constraints were included in the functional analysis because that was the state of the art at the time.

${ }^{8}$ Considering the situation with hindsight, it would have been more appropriate to write a test procedure that confirmed the functionality rather than document the functionality in a traditional requirements document.
} 


\section{Module 4}

Accepted to the18th INCOSE International Symposium, Utrecht, Holland, 2008

Answers. The choices of design for the physical allocation of the CS functionality were to use a minicomputer equivalent to the Hewlett Packard 3000, the Apple Lisa and a Z-80 based S-100 microcomputer ${ }^{9}$. However the sloppy control split function approach reduced the workload of the CS to the point where a Z-80 based S-100 Bus microcomputer ${ }^{10}$ could do the job, saving the project around $\$ 300,000$. Given that the design team had experience programming the Z-80 (in the form of the Intel 8080), and the cost saving (important to a start-up company) the design choice was to go with the Z-80 microcomputer.

The CS software was portioned into Builds using the cataract development methodology (Kasser, 2002a). The Builds were designed to increase automated functionality as more was learned about the operation of the system. Remember this was a 'first of its kind' system. Thus Build 0 would provide the basic operator interface functionality allowing the system to be operated and tested manually. Build 1 would provide the initial automated functionality and future builds would incorporate additional functionally, transferring functions commonly performed by the operator into automatic sequences and any other approved change requests. Since delivery to the customer was dependent on the dates of installation of the mechanical and thermal adjacent SEGS-1 subsystems and not on the date of completion of the software, this approach mitigated the risk of schedule slips due to software related problems and ensured that the CES would perform its basic functionality when delivered. As it happened, the CES was delivered with Build 1 fully operational.

Test. The design decision was supported by experience and calculations that the Z-80 microcomputer was fast enough to do the job.

FRAT applied to the network subsystem. Consider the application of FRAT to the network, subsystem.

Functions. The functions were the communication between the CS and LOCs.

Requirements. The requirements included:

1. Low data rates between the central station and the LOC due to the sloppy algorithm and slow rate of change of both the sun angle and mirror movement

2. Operate no matter what the electromagnetic interference (EMI) environment. The degree of EMI was unknown; there could be thunderstorm induced transients or induction of signals into the underground cable network from nearby local radio stations. As such, from the quantitative STP there was little point in putting any numbers into the requirements.

Answers. The alternative physical design choices to perform the functions to the requirements were coaxial cable-based Ethernet, then in its infancy and hence high risk, or a low data rate twisted pair shield cable, a lower risk option. The low data rates meant that the design need not use Ethernet, nor did it need to compensate for signal deterioration in long coaxial cable runs. There was also a choice in which type of communications protocol to use, the choices being between a polled approach in which the CS interrogated each LOC in turn sequentially and some kind of random access protocol. This choice was independent of the physical design. The design decision allocating the functions to physical components was to use twisted shielded pair cable. In addition, the communications was implemented using:

\footnotetext{
${ }^{9}$ The IBM PC wasn't even a gleam in some engineer's eye at that time, and the Apple Lisa was a not very serious candidate.

${ }^{10}$ With a new colour display card to be designed since one was not available as commercial off the shelf (COTS).
} 


\section{Module 4}

Accepted to the18th INCOSE International Symposium, Utrecht, Holland, 2008

1. A polling protocol in which the central station polled each LOC in turn.

2. The data format was short commands and responses in ASCII at 1200 Baud.

Test. The decisions were verified by a calculation that showed that 1200 Baud was fast enough that the CS could poll and receive a response from each LOC within the predetermined minimum cycle time ${ }^{11}$. This design (functions to physical allocation) approach also allowed a thencommon hand-held ASCII data terminal to be used to troubleshoot communications problems because the technician could bridge the cable and monitor network traffic, or as appropriate, disconnect the network cable and transmit signals to a LOC or the central station and see the response on the hand display.

FRAT applied to power distribution. Applying FRAT to the power distribution subsystem we had:

Functions. The distribution of power to the array of motors and LOCs.

Requirements. The requirements included:

1. The power distribution subsystem shall distribute AC power to the LOCs.

Answers. The physical implementation choices were to distribute power to the LOCs at $110 \mathrm{~V}$, $240 \mathrm{~V}$ or $440 \mathrm{~V}$ using single or three phase power. Factors affecting the choice included the impedance losses in the power cables - the higher the voltage, the lower the losses. The design methodology employed was a problem avoidance approach. Early prototyping of a $240 \mathrm{~V}$ three phase approach had showed that the silicon controlled rectifiers had a high failure rate - a high risk situation. Instead of investigating the causes of these failures, we used continuum thinking to adopt an alternative inherently low risk mixed voltage distribution approach as follows. The bulk of the power would be distributed at $440 \mathrm{~V}$ into the field to strategically located power distribution units (PDU). The loading on the supply phases was balanced by using different phases in different parts of the field. The PDUs would contain a step down 440/110V isolation transformer and transient suppressor. Power from the PDUs would be distributed to a bank of LOCs at $110 \mathrm{~V}( \pm 10 \%)$. This design choice had the following benefits.

1. The use of $110 \mathrm{~V}$ single phase motors which were testable in Israel using a standard 240/110 V step down transformer.

2. The step down transformers in the PDUs also acted as high frequency transient chokes.

3. The $110 \mathrm{~V}$ distribution allowed a single power line to be routed to the LOCS for supplying power to both the motors and the LOC internal electronics.

Test. The feasibility of the decision was verified by analysis at the design phase and subsequent operation at the acceptance test ${ }^{12}$.

\section{The LOC subsystem}

The LOC became the heart of the system and is discussed more thoroughly than the previous

\footnotetext{
${ }^{11}$ Mitigating this risk was the fact that the rate could have been lowered to 300 Baud which would still have been fast enough to provide status information. However, it would have been slow from an operator's point of view.

${ }^{12}$ Would further detailed analyses have changed the geometry of the power distribution and perhaps resulted in lower cable impedance losses? Perhaps? However the customer was satisfied with the architecture and the system was deployed to schedule, so the solution was a correct one. This does not mean that these detailed analyses should not be made sometime in the future when resources become available, and the results of the analyses be used in the design of follow-on systems.
} 
subsystems in this paper. Insights and observations from considering the LOC from various perspectives include:

Operational perspective. Each LOC operates in a self-regulating automatic manner such that the mirror it is controlling, is always pointing at the sun. The operator at the CS or the automatic function in the CS commands and interrogates the LOC in accordance with the operating scenarios alluded to in the system level operational perspective (not provided in this paper).

Functional perspective. Each LOC performs the following functions:

1. Deploy. Upon receipt of a Deploy command, deploys the mirror to the commanded position. Note the position has been determined by the CS to be slightly lower than the calculated sun elevation angle.

2. Search. Upon reaching the deploy position, moves the mirror forwards a few degrees until the sun sensor acquires the sun.

3. Track. Once the sun sensor has acquired the sun, moves the mirror forwards past the sun, stops and waits for the sun to catch up. When the sun catches up, moves it forwards past the sun again and waits, and so on until evening. This function is self-regulating and is independent of the communication link to the CS.

4. Loss of sun. Flywheels as if it was tracking for a short period of time. This function came from the generic perspective; the length of the 'short time' came from the quantitative perspective.

5. Idle. Does not move until told otherwise.

6. Command and control. Moves the mirror and provides oil temperature, and functional mode (deploy, search, track, loss of sun and idle) and position information to the CS upon receipt of the appropriate commands.

Big picture perspective. The LOC subsystem interfaces to a sun sensor, a position sensor and the motor on the adjacent mechanical mirror system as well as a temperature senor in the adjacent heat flow system.

The perspective also points out the risk potential for a mixture of various problems with either adjacent systems or internal subsystems as follows:

- Incorrect interfaces since the mirrors would be coming from Germany.

- Incorrect interfaces since the position sensors would be subcontracted to a Japanese manufacturer.

- The LOCs would be integrated in Jerusalem, from parts purchased both in Jerusalem and California.

- Cabinets and housings were custom made in Israel. Manufacturing tolerances were loose so that mechanical parts were not always interchangeable.

- Power transformers were made in Tel Aviv. The quality as it turned out was excellent.

- Undesired emergent properties. There was no space to build, store and test a full array of more than 600 LOCs in Jerusalem. Anticipating undesired emergent properties to appear after installation in the field ${ }^{13}$, the LOC firmware would be in EPROMS to allow for speedy upgrades. Consequently, there was a configuration risk of not having future fieldinstalled upgrades in-place, and subsequent reporting of already fixed problems.

- The LOCs would contain unique identifiers in chip headers. Each would have to be

\footnotetext{
${ }^{13}$ These emergent properties would be a property of the large array and would not be seen in development.
} 


\section{Module 4}

Accepted to the18th INCOSE International Symposium, Utrecht, Holland, 2008

installed in the designated LOC as part of the system integration in the field. If the headers were to be installed in the wrong LOCs, the CS would experience problems.

- The communication cables would be purchased in the USA, manufactured in California and shipped to the site. This provided the only problem ${ }^{14}$ experienced in the CES. The cables were fitted with push-on connectors for fast and error-free connection. However, the cables used in Jerusalem were flexible and push-on connections stayed in place. The cables purchased in California were not identical and were less flexible. Slowly over time the tension in the cable caused it to pull away from its printed circuit board mounted socket. However, once the cause was determined, the fix was obvious ${ }^{15}$.

Structural perspective. The LOC architecture was that of a standard embedded digital microprocessor with analogue interfaces to the sun sensor, oil temperature sensor, a digital interface to the mirror elevation angle sensor, a serial balanced digital driver interface to the network, and a high-voltage control interface to the motor control circuits. The LOC also contained a Commercial off the Shelf (COTS) alternating current (AC) power supply.

Generic perspective. The LOC is an electronic device in an outdoor desert environment. As such it inherits environmental requirements from that domain.

Continuum perspective. The LOC is not just part of one system, it can be considered as a subsystem of two different systems ${ }^{16}$, namely:

1. The control and electronics system, and

2. The mirror system which contains the LOC, the mirror, the motor that moves the mirror, the mirror elevation angle position sensor, the heat flow elements at the focus of the mirror and the oils temperature sensor in the heat flow element at the center of the mirror.

In addition, subsystem boundaries can be drawn in various ways depending on the interest, for example, the mirrors are part of the SEGS-1mechanical subsystem, and the heat flow elements are part of the SEGS-1 heat flow subsystem.

Temporal perspective. The LOC need not operate 24 hours per day seven days per week since the CS did not generate power at night. However, failures should be readily apparent to the operator so that replacements could be made speedily. Down time is loss of revenue time.

Quantitative perspective. Sun movement is relatively slow as measured in minutes. The rate of collecting telemetry information from the LOCS can be just as slow. Thus exact data rates need not be specified at the system conceptual design review. The test function at the subsystem level will later show that the data rate selected would be/was fast enough for this situation.

Applying FRAT to the LOC. Applying FRAT at the subsystem level to the LOCs we have:

Functions. The functions are as described in the functional and performance perspective above.

Requirements. Some of the LOC requirements are:

1. The mirror position accuracy requirement is inherited from the CES level requirement,

\footnotetext{
${ }^{14}$ The sun sensor was produced by the Physics department who were also responsible for the heat flow subsystem, so technically, the sun sensor problem (discussed later) was a heat flow subsystem problem <grin>.

${ }^{15}$ Tie it down so it wouldn't lift off.

${ }^{16}$ Note that during the design process both views were employed at different times for different purposes.
} 


\section{Module 4}

Accepted to the18th INCOSE International Symposium, Utrecht, Holland, 2008

namely \pm 0.2 degrees.

2. The environmental requirements are inherited from the standard outdoor desert requirements.

3. The data transfer rate for command and control between the CS and the LOC are low due to the sloppy algorithm for controlling the LOC pointing angle.

Answers. Some of the design options for physically implementing the LOC functionality are shown in Figure 3. Design decisions at this level were made between alternative sensors namely:

- Position sensor. The function is sensing the position of the mirror, the physical choice was between:

1. some sort of pulse counter which would count revolutions of the motor from the stow potion and compute the angle reached,

2. an analogue potentiometer in which the resistance would be a function of the angle of elevation and

3. a digital sensor which provided position information in Grey code.

Each option was considered as summarized herein.

- The pulse counter needed some mechanical construction and electronic circuitry and was relatively low risk. The cost was unknown.

- The potentiometer was open to problems in the future due to wear and tear on the track, since the same section would be traversed daily. There was no information on how readily available components behaved under these conditions. The use of high reliability potentiometers from missiles was considered, but their operating conditions were very different since a missile spends most of its time in storage and just a few minutes in flight. In addition the interface would have to be individually calibrated for each sensor in the field after installation. From the temporal perspective, if the sensor degraded in use to the point of replacement, the replacement was expected to be costly and since they could all be expected to fail within the same time frame, the system would have to operate in a degraded mode for some time, while the replacements were manufactured, shipped and installed.

- The optical sensor option was the simplest. The Grey code provided a simple unambiguous position with a digital interface.

The problem of coupling the position sensor to the mirror should the pulse counter not be chosen, was solved by "Rogo", the head of the Mechanical Department who conceptualized hanging a weight on the position encoder shaft so that it acted as a pendulum. Movement of the mirror would change the angle between the pendulum and the fixed point on the mirror. This design could be used with both the potentiometer and the optical sensor. The pendulum and position encoder were encapsulated in an environment proof container that could easily be interfaced to the mirror.

The cost of the selected option was $\$ 300$ per unit, which was not cheap when the total cost to be purchased was 600 or so. However, the simplicity of the design, coupled with the low risk made it the obvious choice at the time. Since the department resources and schedule were limited and an investigation of the alternative choices would take time with no guarantee of lower cost (costs of the alternative sensors and signal processing components, not to mention assembly costs), 


\section{Module 4}

Accepted to the18th INCOSE International Symposium, Utrecht, Holland, 2008

the optical sensor option was chosen and a contract awarded for a few prototypes, and once they had been tested, contract for the manufacture and shipping of the remainder to the installation site was awarded ${ }^{17}$.

- Sun sensor. The sensor was a standard two diode sensor, but there was no specification correlating the incident light to the diode characteristics. This was a major risk because pointing accuracy had a tight tolerance and was critical to the success of the system and there were also no vibration specifications for the mirrors as a result of movement or for any other cause. A "specification free design" was bread boarded, tested and put into service, but there was no firm information as to the design margin.

Test. The sun sensor electronic interface was tested in the laboratory and the embedded software shown to work under all test conditions so the system was passed for deployment. Mind you as it turned out there was a preventable problem with the sun sensors but not in the CES. The sun sensor used a lens to focus the sun onto the pair of photo diodes. During the assembly process the diodes were glued to a base plate with transparent glue. The physics department who were building the sun sensors did not place a manufacturing process requirement that there be no glue on the side of the diode illuminated by the sun. After all, the glue was transparent. A year or so later, they found that the glue slowly became opaque when subjected daily to the very high temperature at the focal point of the lens. This phenomenon resulted in the need to replace all the sun sensors. From a manufacturing perspective, there was little difference in mounting the diodes if the glue could or could not be allowed to cover the face of the diode, just a matter of care and a few extra minutes of time. Nobody in the physics department which designed and produced the sun sensors employed the temporal perspective to ask about possible changes to the characteristics of the glue over long periods of time under high temperature. If the requirement had been placed on the process, not to allow glue on the face of the diode, the characteristics of the glue under the high temperature conditions would not have mattered and the expensive sunsensor replacements would have been avoided (Kasser, 1995). This is an example of introducing an unnecessary failure mode by not utilizing the "don't cares" due to the lack of system thinking. Thus the lesson learned is that if it doesn't make any difference don't do it.

\section{Discussion on the use of FRAT}

The use of the modified FRAT concept has shown how requirements, functional analyses and physical allocation flow from the system to subsystem as the design progresses. One example is that of mirror positioning. The system requirement was to generate power from the sun. This translated to moving and positioning function and a pointing accuracy derived requirement of \pm 0.2 degrees. Further functional decomposition as part of the design process split the moving and positioning function between two physical subsystems the CS and the LOC. The CS functionality determined when to deploy and stow the mirrors, while the LOC functionality kept it positioned to meet the performance requirement. The pointing accuracy requirement was thus implemented in the LOC. Further decisions as to what type of physical element would perform the mirror position sensing function were also shown.

\footnotetext{
${ }^{17} \mathrm{Had}$ the quantity to be manufactured been much greater, a more detailed design and cost analysis might have had to be done to determine the lowest total cost.
} 
Module 4

Accepted to the18th INCOSE International Symposium, Utrecht, Holland, 2008

\section{Lessons learned from the project}

This last part of the paper contains some of the lessons learned from this project. A number of instances are already documented and references are provided in those instances. The lessons learned from this project include:

- KISSE or Keep it simple, systems engineer!

- Focus on people not on process.

- Keep the number of requirements small and pertinent to the mission.

- Requirements are only a communications tool.

- Functional decomposition creates physical architecture and drives design.

- Avoid analysis paralysis.

- View the problem from several perspectives or frames.

- Current 'don't cares' can hurt you in the future.

- Design for test and maintenance.

- Reuse of components simplifies system software as well as hardware.

Consider each in turn.

KISSE or Keep it simple, systems engineer! Was this a system, or a system of systems? System boundaries are in the eye of the beholder (Churchman, 1979) page 91). A LOC was a system in itself containing a mixture of COTS and custom components; the whole array of LOCs was a system. On the other hand, the array of LOCs, communications network and the Central Station was a system. Not only that, but a single LOC and the sensors mounted on its mirror also comprised a system and the whole field was another system. How many systems actually were there? The answer to the two questions is "it depends". System boundaries are drawn for a purpose and represent an abstraction of part of the real world for some purpose. They should be used appropriately (Kasser and Palmer, 2005).

Focus on people not on process. The expertise must be intuitive in the person, not in the manual or in the Standard (Kasser, 2007), in other words, it must be "a philosophy and a way of life" (Hitchins, 1998). A process standard would probably have killed the project. Note in the discussions above, how tradeoff analysis for choosing physical options to meet required functionality were completed only to the point of determining the feasibility of the option, and not down to crossing the last ' $t$ ' and dotting the last ' $i$ '. This was because the systems engineer knew when to stop analyzing and start deciding. This lesson supports the statement by (Frosh, 1969), when he was Assistant Secretary to the United States (of America) Navy which stated: "Systems, even very large systems, are not developed by the tools of systems engineering, but only by the engineers using the tools."

Keep the number of requirements small and pertinent to the mission. There were only two top level requirements. If there is consensus on what the system is to do, don't specify details that can best be left to designers and other stakeholders to determine interactively further down the lifecycle.

Requirements are only a communications tool. If there is a clear understanding of the purpose of the system you may not need them (Kasser, 2002b). The engineers and technicians at LuZ spoke various combinations of Russian, English, French, Romanian and Hebrew, because most were immigrants and there were times when there was no common language in a meeting. Yet in 
Module 4

Accepted to the18th INCOSE International Symposium, Utrecht, Holland, 2008

spite of these communications difficulties, and the lack of written requirements, we knew what we were building and the project succeeded ${ }^{18}$.

Functional decomposition creates physical architecture and drives design. Consider alternate decompositions at design time. The rules for functional decomposition are (Kasser, 1999):

- Minimize coupling and maximize the cohesion.

- Consider the operator as part of the system.

- Use self-regulating subsystems.

- Use railroad buffers for signal passing.

Note use cases and concepts of operations describe functions. For example, we considered various functional options for the mirror positioning functionality and exposed the advantages and disadvantages of each option. The eventual choice was to separate the sun elevation angle calculation function from the mirror pointing functions. This allowed one function to be located in the CS and the other in the LOC. Since the mirror pointing function was designed as a selfregulating function, there was minimal coupling between the two functions (i.e. just the command to deploy, and the necessary angle). It also allowed us to complete the software ahead of schedule.

Avoid analysis paralysis. There is no need to continue an analysis past the point of identification of non-feasibility.

View the problem from several perspectives or frames. Use generic thinking. Someone out there has probably already solved a part of the problem or even your entire problem. This is the core concept in TRIZ (Theory of Inventive Problem Solving) in which it is stated as "Somebody someplace has already solved this problem (or one very similar to it.) Creativity is now finding that solution and adapting it to this particular problem" (Barry, et al., 2007). However, you may have to redefine your problem to use that solution to avoid unnecessary complicating the situation. The generic perspective helps to identify candidate solutions for pattern matching to your situation.

Current 'don't cares' can hurt you in the future. Don't add unnecessary parts even if they appear not to make a difference at design time. The sun sensor glue was an unnecessary part when placed in front of the photo diode as discussed above.

Design for test and maintenance. Test and integration needs to be considered at production process design time as well as product design time. Add test points to both the hardware and software (Kasser, 1997). Embedded software in-circuit emulation will not find all problems. Choose a software architecture such as a 'state machine' which can be thoroughly tested and use buffers to store data being passed between functions for simplifying testing.

Reuse of components simplifies system software as well as hardware. Reuse of the mirror pointing algorithm in each LOC simplified the CES design. This was an object-oriented approach inherited from hardware expertise.

\section{Meta-lessons learned}

Now just because these lessons were learned from this system, they should not be allied indiscriminately to all future systems. Note, there are two kinds of meta-lessons to be learnt,

\footnotetext{
${ }^{18}$ Delivered on time within budget and performed to requirements.
} 


\section{Module 4}

Accepted to the18th INCOSE International Symposium, Utrecht, Holland, 2008

- Some of these lessons apply to all projects, and

- Some of these lessons apply to some projects.

Think before you apply them, and ask yourself the following two questions.

- Why did it work here?

- What is different about your project?

\section{Summary}

This paper has documented the LuZ SEGS-1 system design process in the form of the FRAT views demonstrating the intertwined relationships between requirements, functions and their allocation to components at a lower level of system decomposition. The paper provided examples of alternative choices, discussed them and document the choice with the reasons for selection. Several lessons learned from the project were also provided.

\section{Author's biography}

Prof Kasser has been a practicing systems engineer for 35+ years and an academic for about 10 years. He is an INCOSE Fellow, the author of "A Framework for Understanding Systems Engineering" and "Applying Total Quality Management to Systems Engineering" and many INCOSE symposia papers. He is a recipient of NASA's Manned Space Flight Awareness Award (Silver Snoopy) for quality and technical excellence for performing and directing systems engineering and other awards.

He holds a Doctor of Science in Engineering Management from The George Washington University, and is a Certified Manager. He has also served as President of INCOSE Australia and Region VI Representative to the INCOSE Member Board.

He is a principal at the Right Requirement Ltd. He gave up his positions as a Deputy Director and DSTO Associate Research Professor at the Systems Engineering and Evaluation Centre at the University of South Australia in early 2007 to move back to the UK to develop the world's first immersion course in systems engineering as a Leverhulme Visiting Professor at Cranfield University.

\section{References}

Barry, K., Domb, E. and Slocum, M. S., TRIZ - What Is TRIZ?, 2007, http://www.trizjournal.com/archives/what_is_triz/, last accessed 31 October 2007

Churchman, C. W., The Systems Approach and its Enemies, Basic Books, Inc., New York, 1979.

Donaldson, S. E. and Siegel, S. G., Cultivating Successful Software Development A Practitioner's View, Prentice Hall PTR, Upper Saddle River, New Jersey, 1997.

Frosh, R. A., "A new look at systems engineering", IEEE Spectrum, Vol. (1969), no. September.

Hitchins, D. K., "Systems Engineering...In Search of the Elusive Optimum", proceedings of Fourth Annual Symposium of the INCOSE-UK, 1998.

Kasser, J. E., "A Distributed Control System for a Solar Collector Field", proceedings of Energy 84, Tel Aviv, Israel, 1984.

Kasser, J. E., Applying Total Quality Management to Systems Engineering, Artech House, Boston, 1995.

Kasser, J. E., "Yes Virginia, You Can Build a Defect Free System, On Schedule and Within Budget", proceedings of INCOSE 7th International Symposium, Los Angeles, CA, 1997. 
Module 4

Accepted to the18th INCOSE International Symposium, Utrecht, Holland, 2008

Kasser, J. E., "Using Organizational Engineering to Build Defect Free Systems, On Schedule and Within Budget", proceedings of PICMET, Portland OR., 1999.

Kasser, J. E., "Writing Requirements for Flexible Systems", proceedings of INCOSE-UK Spring Symposium, 2001.

Kasser, J. E., "The Cataract Methodology for Systems and Software Acquisition", proceedings of SETE 2002, Sydney Australia, 2002a.

Kasser, J. E., "Does Object-Oriented System Engineering Eliminate the Need for Requirements?" proceedings of the 12th International Symposium of the International Council on Systems Engineering, Las Vegas, NV., 2002b.

Kasser, J. E., "Eight deadly defects in systems engineering and how to fix them", proceedings of the 17th International Symposium of the INCOSE, San Diego, CA, 2007.

Kasser, J. E., "Applying systems thinking and aligning it to systems engineering", proceedings of submitted to the INCOSE International Symposium, Utrecht, Holland, 2008.

Kasser, J. E., Kaffle, S. and Saha, P., "Applying FRAT to improve systems engineering courseware: Project Review", proceedings of SEEC Research Group, SESA-South Australia and INCOSE-Australia joint meeting, Adelaide, 2007.

Kasser, J. E. and Palmer, K., "Reducing and Managing Complexity by Changing the Boundaries of the System", proceedings of the Conference on Systems Engineering Research, Hoboken NJ, 2005.

Mar, B. and Morais, B., "FRAT A Basic Framework for Systems Engineering", proceedings of 12th annual International Symposium of the International Council on Systems Engineering, Las Vegas, NV., 2002.

Mar, B. W., "Systems Engineering Basics", Systems Engineering: The Journal of INCOSE, Vol. 1 (1994), no. 1, 7-15. 